\title{
Research of FFT Improved Asynchronous Sample Arithmetic in Harmonic Component Detection
}

\author{
XiaoLing Yan ${ }^{1, a^{*}}$, LiMing Wang ${ }^{2, b}$ \\ Electric and Information College, Naval University of Engineering, \\ 430033 Wuhan, Hubei, China \\ ${ }^{1,2}$ Electric and Information College, Naval University of Engineering, 430033,Wuhan, Hubei, China \\ ayanx10213@163.com, bicesoar@163.com
}

\begin{abstract}
Keywords: harmonic pollution, spectrum leakage, fence effect
Abstract. Along with the diversification and complication development of electric equipment in warship, the harmonic pollution in power system is more and more seriously, and the harmonic detection technology increasingly turns into a very important subject. FFT arithmetic is an usual method to detect harmonic component, but this method is often accompanied with spectrum leakage and fence effect which can bring measurement error. The article analyses spectrum character of different window functions, compares merits of different interpolation methods, and then improves FFT arithmetic and defines detection index. The experiment proves that the improved method gets better result on detecting harmonic signal which fundamental frequency is $49.8 \mathrm{~Hz}-50.2 \mathrm{~Hz}$.
\end{abstract}

\section{Introduction}

As warship power system develops rapidly, more and more electric devices are applied in it. In these devices, nonlinear loads, such as AC controller, electric motor, transformer, inverter, chopper, medium voltage DC power distribution and etc., occupies a large proportion. These nonlinear devices can produce serious harmonic pollution in power system which will descend power system quality and even damage some accurate equipment. Therefore, harmonic component detection technology is very important for power system being in safe and stable operation.

Now, the harmonic component detection mostly uses method based on FFT arithmetic. If calculate periodic signal by FFT, there must add a window function to cut off the signal and take one or several complete cycles. This action can certainly bring spectrum leakage. When measuring electrical quantity, if sample frequency is not equal to fundamental frequency, the result is leakage spectrum rather than right value. This situation is called fence affect. Because of the error of calculation and phase-angle, the method is fit for harmonic detection anymore when load changes suddenly. The article compares rectangular window, Haiming window and Hanning window, chooses appropriate window D-value, and improves the FFT arithmetic which can be better for harmonic detection.

\section{Index of Harmonic Component Detection}

In warship power system, the sinusoidal quantity which has the same frequency with power source is fundamental component. In addition, there are more and more high order harmonic components in it with the increase of complexity. The direct influence to power system is the cyclical distortion of current and voltage waves. As shown in Fig.1 and Fig.2, one is normal voltage wave without harmonic component, and the other has 3,5,7,9 harmonic components. 


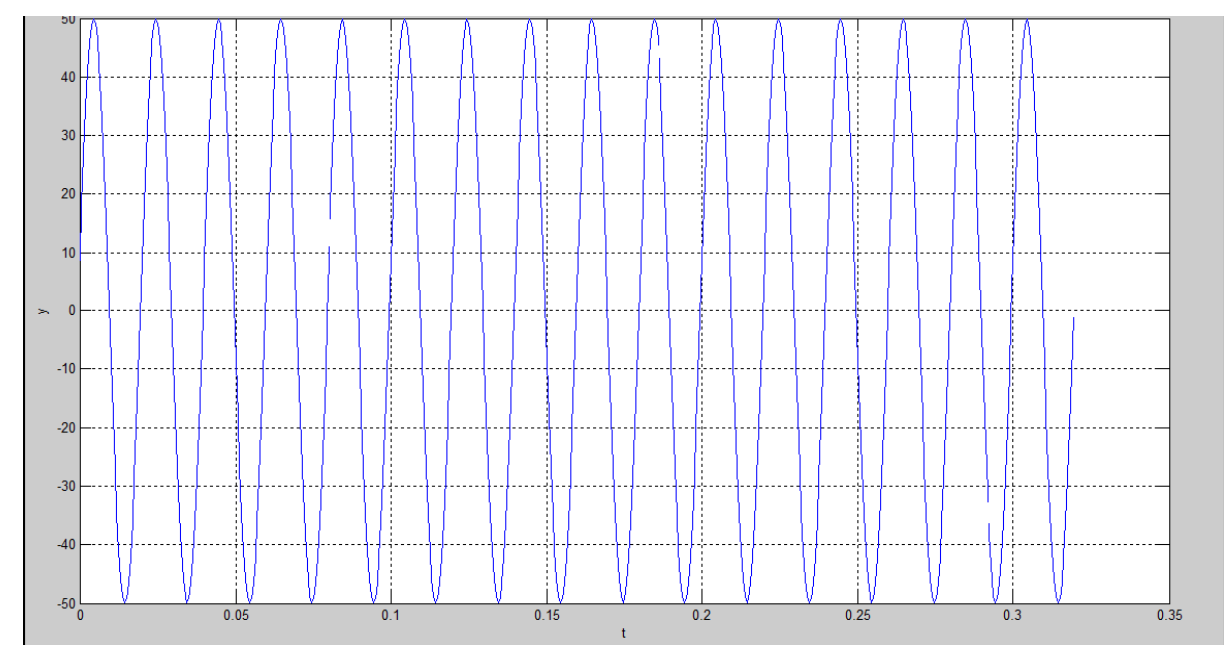

Fig.1 voltage wave without harmonic component

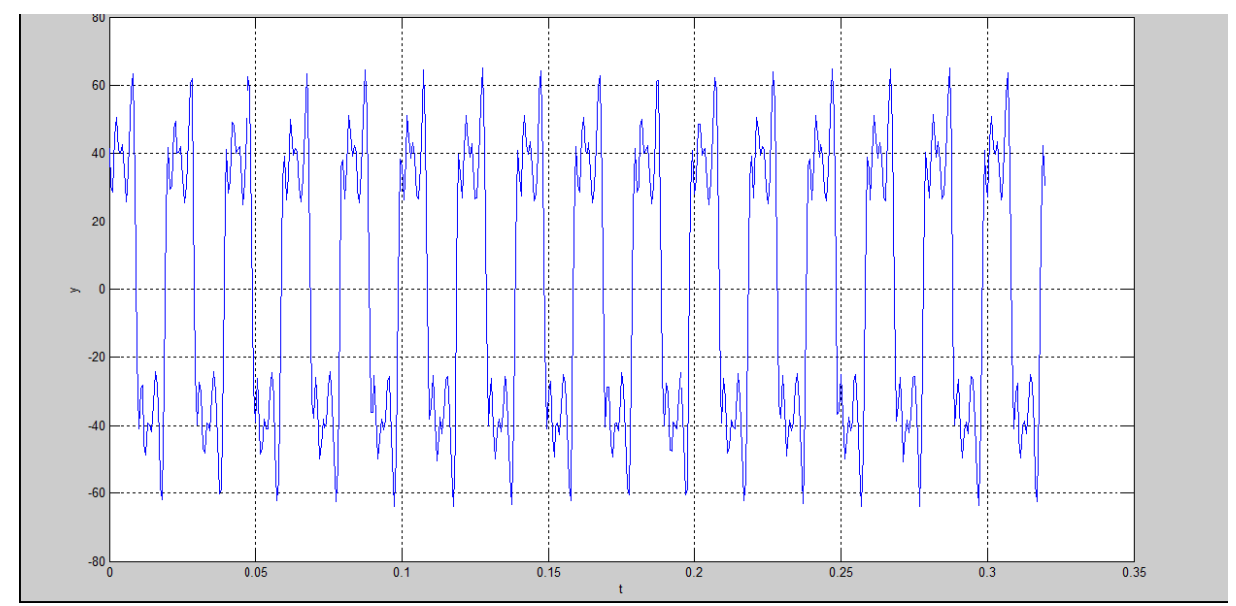

Fig.2 voltage wave with $3 、 5 、 7 、 9$ harmonic components

The level of distortion wave deviating sinusoidal waveform is usually indicated by distortion rate. This rate value is the percentage of the root sum square of all harmonic waves' effective values and fundamental wave effective value. In order to quantitatively said the distortion degree of the sinusoidal waveform of power system, it is expressed by every harmonic component content and total harmonic distortion rate (THD). The distortion rate of voltage sine wave is defined as follow:

$$
D F U=\frac{100 \sqrt{\sum_{n=2}^{\infty} U_{n}^{2}}}{U_{1}} \%
$$

In formula (1), $U_{1}$ is rated fundamental voltage, which can be expressed by effective value of fundamental voltage.

In engineering, the $\mathrm{n}^{\text {th }}$ harmonic content $\mathrm{DFU}_{\mathrm{n}}$ is the percentage of the effect value of the $\mathrm{n}^{\text {th }}$ harmonic voltage $U_{n}$ and the fundamental wave effect value $U_{1}$.

$$
D F U_{n}=\frac{100 U_{n}}{U_{1}} \%
$$

It should be known that the harmonic components in distortion wave are not different in various cycles. Its value is maybe random variation and differs greatly. International Conference on Large High Voltage Electric System (CIGRE) suggests that when measure and calculate harmonic waves' effective value, it should be the average in 5 seconds to distinguish transient phenomena. 


\section{Principle of Harmonic Analysis}

French scientist Fourier said, an arbitrary function $x(\mathrm{t})$ can be decomposed into an infinite number of different frequency sinusoidal signals. This is the fundamental of harmonic analysis. After Fourier analysis is involved, FFT method became an important instrument for harmonic analysis.

\section{Discrete Fourier transform}

Discrete Fourier transform is one the most basic and common arithmetic in digit signal processing. In engineering, signals are usually defined as a discrete and finite sequence in time domain and frequency domain. Thus, Fourier transform must be on real discrete points of $f(\mathrm{t})$ so that signal's frequency analysis and other processing then can be operated in practice.

Given discrete time series $\left(x_{0}, x_{1}, x_{2}, \ldots, x_{\mathrm{n}}\right)$, this series is Absolutely summable as expressed by formula(3)

$$
\sum_{n=0}^{N-1}|x(n)|<\infty
$$

Then its discrete Fourier transform (DFT) and inverse transform are defined as formulas below.

$$
\begin{aligned}
& X(k)=\sum_{n=0}^{N-1} x(n) W_{N}^{n k} \quad(n=0,1 \ldots N-1) \\
& x(n)=\frac{1}{N} \sum_{n=0}^{N-1} X(k) W_{N}^{-n k} \quad(k=0,1 \ldots N-1)
\end{aligned}
$$

In these formulas, $W_{N}=e^{-j \frac{2 \pi}{N}}$ 。

From analysis above, it can be known that in DFT operation, no matter multiplication or addition, the calculational quantity is in proportion to $\mathrm{N}^{2}$. When $\mathrm{N}$ is large, the calculational quantity is large and the time cost is huge. Because of this issue, DFT can't be widely used in engineering application.

\section{Fast Fourier Transform Arithmetic(FFT)}

In order to overcome the disadvantage of DFT, J.W.Cooley and J.W.Tukey proposed Fast Fourier Transform which is called FFT in 1965. FFT is not a new method. It is just an improved faster arithmetic of DFT. It takes advantage of the periodicity, symmetry and orthogonality of exponential function which can greatly decrease the calculational quantity of DFT.

Through analysis on $W_{N}^{n k}=e^{-j \frac{2 \pi}{N} n k}$, we can get some characteristics:

(1)Symmetry: $\left(W_{N}^{n k}\right)^{*}=W_{N}^{-n k}$

(1)Periodicity: $W_{N}^{n k}=W_{N}^{(N+n) k}=W_{N}^{(N+k) n}$

(2)Reducibility: $W_{N}^{n k}=W_{m N}^{m n k}=W_{N}^{(N+k) n}$

Based on the characters above, some items in DFT can be combined. Its operation can also be resolved into several smaller DFT which has a minor value of $\mathrm{N}$. This way can reduce amount of calculation. The development of FFT is just about this basic theory. Because of its superiority of calculation time cost, it is widely used in practice.

\section{FFT Improved Method with Window Function and Interpolation}

When calculate harmonic waves in power system by FFT method, the voltage and current signals must be cut off to one or several complete cycles. This will inevitably produce spectrum leakage and disturb between different spectrums. Because the frequency in power system usually fluctuates, the signals obtained by cutting off maybe is not complete cycles when sample frequency is not equal to the number of power system frequency. In this case, the calculation result is the leakage spectrum which is called fence effect. The fence effect can produce errors to harmonic frequency, amplitude and phase.

The improved FFT method with window function and interpolation can solve this problem. This method is an asynchronous sample way. By this way, signals are sampled by fixed sampling 
frequency and the leakage spectrum will be modified by window function's spectrum characteristic. The actual spectrum of the signal can be obtained at last which has little error. But different window function has different influence to result.

\section{Chosen of window functions}

for some given signal, the chosen of window function is very important. The window function is wider, it has stronger power to restrain nose wave and it is narrower, the resolution ratio is higher. The best way to choose window function should be as follow:

(1)The main lobe of function is narrow as far as possible. As a result, the energy will mainly concentrate in main lobe and higher frequency resolution will be gotten when analyzing.

(2)The height of side lobe is small as far as possible and it rapidly attenuates with frequency so that to reduce leakage distortion.

But the window function as above can't be found. For example, rectangular window has a narrowest main lobe, but its side lobe is very big. So the chosen method has to be considered many elements. The article found out that Hanning window is more fit to do spectrum analysis by a lot of experiments. This window can entirely separate the frequency components of the multi frequency signal.

Hanning window is also called raised cosine window. It can be regarded as a spectrum sum of 3 rectangle windows. It can make side lobe offsets each other and eliminates high-frequency interference. The price of smaller side lobe in Hanning window is that the main lobe adds to $8 \pi / \mathrm{N}$, the leakage spectrum of primary spectrum is a half of main lobe that are twice of rectangle window. so, in order to separate fundamental wave and high order harmonic component, the length of Hanning window is at least 2 cycles of fundamental wave.

There are two expressions about Hanning window that are shown as follow:

$$
\begin{aligned}
& \operatorname{hann}(N)=\sin \left(p i^{*}(0: N-1) /(N-1)\right)^{2} \\
& \operatorname{hanning}(N)=\sin \left(p i^{*}(1: N) /(N+1)\right)^{2}
\end{aligned}
$$

If the frequency of fundamental wave $\mathrm{f}_{0}=50 \mathrm{~Hz}$, the signal is shown like formula(8). This signal has 3, 5, 7, 9 harmonic components and their amplitudes and phases are different. If the sampling frequency $\mathrm{Fs}=1600 \mathrm{~Hz}$, sampling points $\mathrm{N}=1024$, its ideal indexes should be as table 1 .

$$
\begin{gathered}
x=380 \sin \left(2 \pi f_{0}+\frac{10 \pi}{180}\right)+20 \sin \left(2 \pi 3 \pi f_{0}+\frac{25 \pi}{180}\right)+25 \sin \left(2 \pi 5 \pi f_{0}+\frac{120 \pi}{180}\right) \\
+10 \sin \left(2 \pi 7 \pi f_{0}+\frac{150 \pi}{180}\right)+5 \sin \left(2 \pi 9 \pi f_{0}+\frac{120 \pi}{180}\right) \\
\text { Table.1 ideal indexes of original signal }
\end{gathered}
$$

\begin{tabular}{|c|c|c|c|}
\hline components Index & Frequency & Amplitude & phase \\
\hline Fundamental wave & 50 & 380 & 10 \\
\hline $3^{\text {th }}$ harmonic & 150 & 20 & 25 \\
\hline $5^{\text {th }}$ harmonic & 250 & 15 & 100 \\
\hline $7^{\text {th }}$ harmonic & 350 & 10 & 150 \\
\hline $9^{\text {th }}$ harmonic & 450 & 5 & 120 \\
\hline
\end{tabular}
table.3.

When the signal goes through Hann window and Hanning window, its indexes is in table. 2 and

Table. 2 Hann window index

\begin{tabular}{|c|c|c|c|}
\hline components Index & Frequency & Amplitude & phase \\
\hline Fundamental wave & 49.9939 & 379.2581 & 10.0003 \\
\hline $3^{\text {th }}$ harmonic & 149.9941 & 19.9615 & 24.9890 \\
\hline $5^{\text {th }}$ harmonic & 249.9939 & 14.9708 & 99.9985 \\
\hline $7^{\text {th }}$ harmonic & 349.9939 & 9.9805 & 149.9996 \\
\hline $9^{\text {th }}$ harmonic & 449.9939 & 4.9903 & 119.9973 \\
\hline
\end{tabular}


Table.3 Hanning window index

\begin{tabular}{|c|c|c|c|}
\hline components Index & Frequency & Amplitude & phase \\
\hline Fundamental wave & 50.0061 & 380.7438 & 9.2959 \\
\hline $3^{\text {th }}$ harmonic & 150.0059 & 20.0000 & 24.3071 \\
\hline $5^{\text {th }}$ harmonic & 250.0061 & 15.0000 & 99.2977 \\
\hline $7^{\text {th }}$ harmonic & 350.0061 & 10.0000 & 149.2967 \\
\hline $9^{\text {th }}$ harmonic & 450.0061 & 5.0000 & 119.2990 \\
\hline
\end{tabular}

From table. 2 and table.3, it can be seen that after signal goes through Hann window, its frequency and amplitude values are alwayse smaller. However, they are alwayse bigger after Hanning window. the difference between hann and hannning window is the first point and the last point. The first point of hann window is $\sin (0)$ and the last one is $\sin (\pi)$. The first point of hanning window is $\sin (\pi /(N-1))$ and the last one is $\sin (\pi N /(N-1))$. The rest of these two windows are 0 . As a result, the result with hanning is bigger, and it's smaller with hann.

According to the property above, the article improves hanning window. the modified window function is like formula 9.

$$
\operatorname{han}(N)=(\operatorname{hanning}(N)+\operatorname{hann}(N)) / 2
$$

After goes through the improved Hanning window, the result index is in table. 4 .

Table.4 improved Hanning window result index

\begin{tabular}{|c|c|c|c|}
\hline components Index & Frequency & Amplitude & phase \\
\hline Fundamental wave & 50.0061 & 380.7438 & 9.6478 \\
\hline $3^{\text {th }}$ harmonic & 150.0000 & 20.0386 & 24.6478 \\
\hline $5^{\text {th }}$ harmonic & 250.0000 & 15.0292 & 99.6478 \\
\hline $7^{\text {th }}$ harmonic & 350.0000 & 10.0195 & 149.6478 \\
\hline $9^{\text {th }}$ harmonic & 450.0000 & 5.0097 & 119.6478 \\
\hline
\end{tabular}

The result proves that the improved method has better effection on the signal frequency and amplitude.

\section{Chosen of interpolation method}

Interpolation method is an estimation method when the sample process is not asynchronous which sampling cycle is not integer times of signal cycle and sampling point is not equal to spectrum point. In this case, the spectrum points should be estimated by interpolation method. The article adjudges interpolation method by degree of approximation indexes. The indexes include degree of approximation of frequency $\mathrm{Pf}$, amplitude $\mathrm{Pa}$ and phase $\mathrm{Pq}$. These indexes defines as follow:

$$
\begin{gathered}
P_{f}=\frac{100\left(F_{i}-F\right)^{2}}{F} \% \\
P_{a}=\frac{100\left(A_{i}-A\right)^{2}}{A} \% \\
P_{q}=\frac{100\left(Q_{i}-Q\right)^{2}}{Q} \%
\end{gathered}
$$

The original signal is handled by different interpolation methods and the results are in table.5. 
Table. 5 results by different interpolation methods on original signal

\begin{tabular}{|c|c|c|c|c|c|c|}
\hline components Index & Frequency & Amplitude & phase & Pf & $\mathrm{Pa}$ & $\mathrm{Pq}$ \\
\hline \multirow{5}{*}{ No interpolation } & 46.8750 & 379.9514 & 9.6458 & $19.5313 \%$ & $0.006215 \%$ & $1.2546 \%$ \\
\hline & 146.8750 & 20.0687 & 24.6448 & $6.5104 \%$ & $0.0236 \%$ & $0.5047 \%$ \\
\hline & 246.8750 & 14.9801 & 99.8169 & $3.9063 \%$ & $0.0026 \%$ & $0.0335 \%$ \\
\hline & 346.8750 & 9.9850 & 149.2133 & $2.7902 \%$ & $0.0023 \%$ & $0.4126 \%$ \\
\hline & 44.8750 & 5.0192 & 118.9408 & $2.1701 \%$ & $0.0074 \%$ & $0.9349 \%$ \\
\hline \multirow{5}{*}{ Triangle interpolaion } & 43.7498 & 380.0277 & 9.6683 & $78.1300 \%$ & $0.00209 \%$ & $1.1002 \%$ \\
\hline & 143.7477 & 20.0349 & 24.9437 & $26.0608 \%$ & $0.0061 \%$ & $0.0127 \%$ \\
\hline & 243.7455 & 15.0479 & 99.8443 & $15.6475 \%$ & $0.0153 \%$ & $0.0242 \%$ \\
\hline & 343.7395 & 10.0254 & 150.2398 & $11.1982 \%$ & $0.0065 \%$ & $0.0383 \%$ \\
\hline & 443.7540 & 50381 & 11.9781 & 8.6694 & 0.020 & $0.8702 \%$ \\
\hline \multirow{5}{*}{ Singher interpolation } & 49.9999 & 379.9738 & 9.6690 & $2.0 * 10^{-8} \%$ & $0.001806 \%$ & $1.0956 \%$ \\
\hline & 149.9976 & 20.0010 & 24.8084 & $3.8 * 10^{-6} \%$ & $5.0 * 10^{-6} \%$ & $0.1468 \%$ \\
\hline & 250.0013 & 15.0416 & 99.6461 & $6.8^{*} 10^{-7} \%$ & $0.0115 \%$ & $0.1252 \%$ \\
\hline & 349.9976 & 10.0052 & 149.8671 & $1.6^{*} 10^{-6} \%$ & $0.02704 \%$ & $0.0118 \%$ \\
\hline & 450.0022 & 4.9763 & 120.3435 & $1.1 * 10^{-6} \%$ & $0.0112 \%$ & $0.0983 \%$ \\
\hline
\end{tabular}

In table.5, there are 512 sampling points. When using non-interpolation method and triangle interpolation method, the value of $\mathrm{Pf}$ is bigger which means that the frequency degree of approximation is poor and it can hardly precisely estimate the spectrum point. However, the Singher function has smaller Pf which can satisfy the demand. The experiment proves that the improved FFT method in this article which uses improved Hanning window and is optimized by Singher interpolation method is preferable for power system's harmonic detection.

\section{References}

[1] WeiMing Ma. The direction of the development of marine power [J]. Journal of Navel University of Engineering, 2002， 14(6)

[2] WeiMin Yin, Hua OuYang, Mei Qian. Digital Signal Process. Machinery Industry Press

[3] FuSheng Zhang, ZhongXing Geng, YaoZhong Ge. FFT Algorithm with high accuracy for harmonic analysis in power system [J]. Proceedings of the Chinese society for electrical engineering. 1999

[4] CaiJun Qi, LongDao Chen. High accuracy estimation of electric harmonic parameters by using the interpolated FFT algorithm[J]. Journal of ZheJiang University, 2003 37(1)

[5] RunYu Lv. High harmonic wave analysis in power system. China Electric Power Press. 1998. 5

[6] HangSheng Li, Dan Chen. Research of the Window function in frequency analysis. Micro Computer Information. 2008 